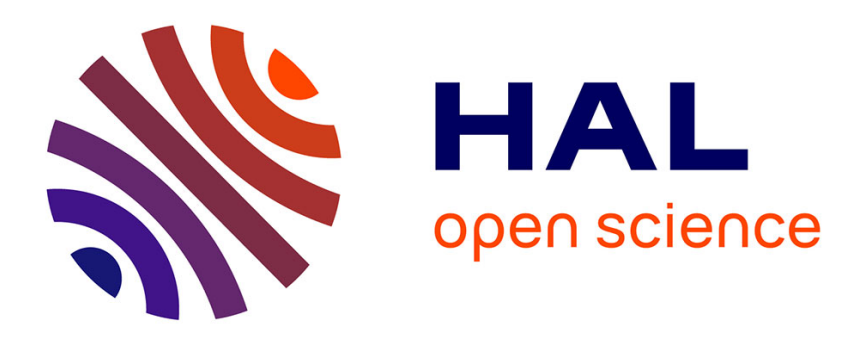

\title{
L'aquaculture dans les mers tempérées
}

Albert Lucas, Nadine Lucas

\section{To cite this version:}

Albert Lucas, Nadine Lucas. L'aquaculture dans les mers tempérées. Norois, 1980, 106, pp. 237-250. 10.3406/noroi.1980.3888 . hal-01173959

\section{HAL Id: hal-01173959 \\ https://hal.science/hal-01173959}

Submitted on 8 Jul 2015

HAL is a multi-disciplinary open access archive for the deposit and dissemination of scientific research documents, whether they are published or not. The documents may come from teaching and research institutions in France or abroad, or from public or private research centers.
L'archive ouverte pluridisciplinaire HAL, est destinée au dépôt et à la diffusion de documents scientifiques de niveau recherche, publiés ou non, émanant des établissements d'enseignement et de recherche français ou étrangers, des laboratoires publics ou privés. 


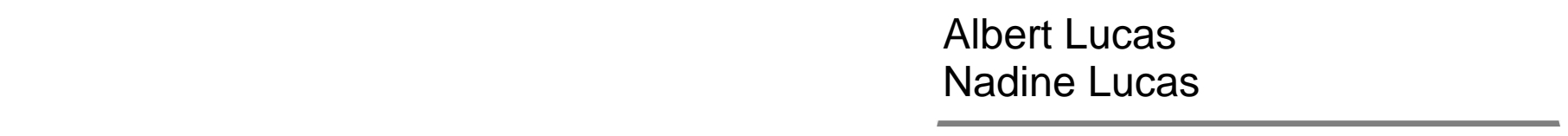

\title{
L'aquaculture dans les mers tempérées
}

\author{
In: Norois. N¹06, 1980. pp. 237-250.
}

\begin{abstract}
Résumé
Les productions marines par culture pouvaient être évaluées en 1977 à 2,2 millions de tonnes (dont 1 million d'algues) tandis que les captures par pêche s'élevaient à 60,5 millions. La mariculture est en pleine évolution technologique et s'est considérablement diversifiée dans les vingt dernières années, notamment au Japon et, dans une moindre mesure, en Europe et aux Etats-Unis. Cependant les tonnages annuels de l'aquaculture dans les mers tempérées, ne progressent guère depuis vingt ans, sauf dans certains secteurs au Japon et en Corée du Sud. Les causes de cette stagnation sont surtout les épidémies et les pollutions qui font chuter les cultures traditionnelles, le manque d'espace et les difficultés techniques qui gênent la progression des nouvelles.
\end{abstract}

\section{Abstract}

Marine culture, in the year 1977, can be evaluated at 2.2 millions tons (1 million tons for algae), while the catches by fishing amounted to 60.5 millions tons. Mariculture is in full technical evolution and was considerably diversified in the last twenty years, especially in Japan and, to a lesser degree, in Europe and U.S.A. However the annual production of aquaculture in temperate seas did not increase during the last twenty years, except in some areas in Japan and South Korea. The causes of this stagnation were on one hand épidémies and pollutions which determined a decrease in traditional cultures, on the other hand the lack of space and under development in technics which retard the progress of new cultures.

Citer ce document / Cite this document :

Lucas Albert, Lucas Nadine. L'aquaculture dans les mers tempérées. In: Norois. Nº106, 1980. pp. 237-250.

http://www.persee.fr/web/revues/home/prescript/article/noroi_0029-182X_1980_num_106_1_3888 


\title{
L'aquaculture dans les mers tempérées
}

\author{
par Albert LUCAS \\ Faculté des Sciences. BREST \\ et Nadine LUCAS \\ INSEAD, FONTAINEBLEAU
}

On entend, par aquaculture, toute culture ou élevage de plantes et d'animaux aquatiques, intervenant pendant tout ou partie de leur cycle de développement. Lorsque le milieu aquatique est la mer, on précise " aquaculture marine» ou "mariculture ». Cependant, notamment en économie et en politique, le terme aquaculture a pris un sens restreint - en même temps que prestigieux - pour ne désigner que les méthodes nouvelles d'élevage en mer, sans prendre en compte les cultures traditionnelles telles que l'ostréiculture et la mytiliculture. Nous n'adopterons pas ce point de vue, d'une part parce que l'aquaculture nouvelle est encore faiblement productrice, d'autre part parce que les techniques récentes et anciennes ont, dans bien des cas, tendance à s'enrichir mutuellement et donc à se confondre. Parlant de l'aquaculture dans son sens large, nous examinerons cependant successivement la traditionnelle et la nouvelle.

Notre étude est limitée aux mers tempérées, car c'est là que la majorité des techniques aquacoles ont été créées et que les productions de culture sont importantes. Comme cette activité est uniquement côtière, c'est en énumérant les pays concernés que nous définirons le mieux notre champ d'investigation. Pour l'hémisphère nord: les pays maritimes d'Europe et ceux riverains de la Méditerranée et de la Mer Noire (environ 30); la Corée et le Japon, les Etats-Unis et le Canada. Pour l'hémisphère sud : le Chili et l'Argentine, le sud de l'Australie et la Nouvelle-Zélande. Dans cette longue liste nous n'aurons à citer que quelques pays, remarquables soit par leur production soit par leur spécialisation. 


\section{I. - L'AQUACULTURE TRADITIONNELLE.}

Les cultures traditionnelles de coquillages sont caractérisées par le fait que ce qui correspond à la semence, le « naissain », est capté en mer sur des collecteurs appropriés (ex : tuiles chaulées pour les huîtres). Cet approvisionnement en semence, réalisé en milieu naturel, risque d'être tributaire des conditions écologiques et climatiques et donc de peser lourdement sur la production finale. C'est effectivement le cas pour les huîtres, mais pas pour les moules, qui sont tellement prolifiques que le naissain ne manque jamais.

Les cultures ont lieu sur des concessions soit sur l'estran, soit en zone non découvrante. Dans le premier cas, on pratique des cultures "à plat » sur sol et des cultures «surélevées" sur des supports métalliques où sont fixés des sacs ou des caisses; dans le second cas des cultures à plat dites en « eaux profondes » et des cultures « suspendues » sur madriers ou sur cordes. La productivité est meilleure quand on passe du sol à la surélévation et de celle-ci à la suspension.

\section{A) Les huîtres.}

Ces mollusques bivalves sont de loin les espèces marines les plus cultivées. En 1977, on pouvait évaluer à $650.000 \mathrm{t}$ (coquille comprise) la production mondiale d'huîtres cultivées, celle des huitres pêchées étant de l'ordre de $80.000 \mathrm{t}$.

On distingue deux sortes d'huîtres comestibles : les « creuses " appartenant au genre Crassostrea, qui est ovipare et les «plates " au genre Ostrea qui est larvipare. Dans ce cas, les œufs ne sont pas relâchés dans la mer, mais conservés entre les valves (huitres "laiteuses") où ils évoluent en larves (huîtres «plombées »). Les larves sont expulsées lorsqu'elles atteignent $0,2 \mathrm{~mm}$. Sur le plan gustatif, les huîtres plates sont plus appréciées que les creuses, ce qui se traduit dans les prix. Ex.: en France, en 1978, les prix moyens au kilo ont été de 17,59 F et 5,96 F respectivement.

L'huître japonaise Crassostrea gigas, qui est cultivée non seulement en Extrême-Orient, mais aussi en Europe et en Amérique du Nord, est la plus productive. En 1977, on pouvait évaluer son tonnage à environ 500.000 t, dont 213.000 au Japon, 161.000 en Corée, 83.000 en France, 22.000 aux Etats-Unis et 3.000 au Canada. Depuis 1971 en France, l'huître portugaise Crassostrea angulata, victime de la "maladie des branchies ", a totalement disparu et a été remplacée par la gigas (tabl. I). La portugaise encore présente au Portugal n'y a produit que $215 \mathrm{t}$ en 1975.

L'huître américaine Crassostrea virginica est cultivée sur la côte atlantique du Canada et des Etats-Unis. Elle est plus réputée que la gigas (cultivée sur la côte pacifique). La production a atteint $120.000 \mathrm{t}$. en 
1977, dont 119.000 aux Etats-Unis. Dans l'est de l'Australie, c'est une espèce autochtone Crassostrea commercialis qui a été mise en valeur. La production dépassait légèrement les $10.000 \mathrm{t}$ en 1977. Elle est de cet ordre depuis plusieurs années.

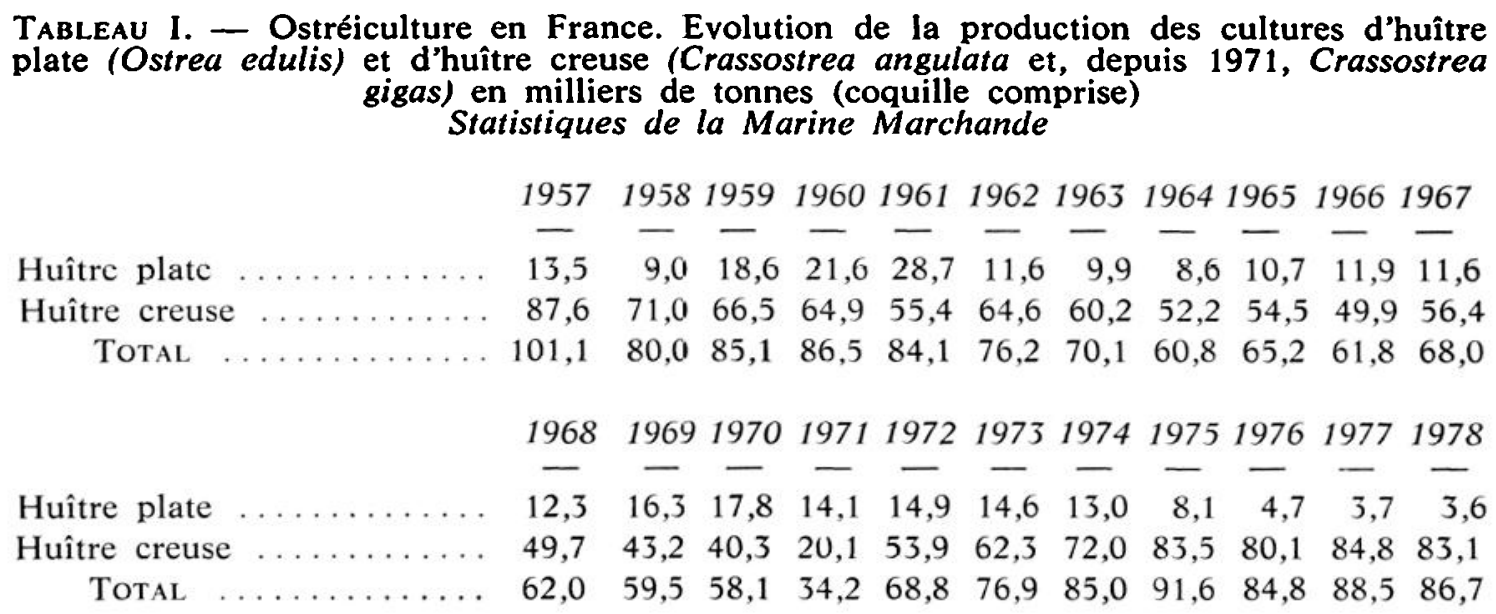

Parmi les espèces d'huîtres plates, il n'y en a qu'une seule qui soit cultivée à grande échelle : Ostrea edulis, l'huître plate européenne. La production française, qui est de loin la plus importante sur le plan mondial, a subi deux chutes spectaculaires (tabl. I): l'une, brutale, due aux grands froids de l'hiver 1962-1963, l'autre, plus lente mais plus ample, due à l'extension progressive de la «maladie de la glande digestive » dans les années 1970. Cette épidémie a touché un peu plus tard 'l'Espagne (2.100 t en 1974, 560 en 1977). Outre la France, seule la Hollande dépassait le millier de tonnes en 1977, aussi peut-on estimer que la production mondiale de cette espèce en 1977 a été de l'ordre de $5.000 \mathrm{t}$. C'est peu pour la plus réputée des huittres, celle que tous les pays voudraient promouvoir.

Au niveau technique, l'ostréiculture traditionnelle s'est considérablement transformée depuis une vingtaine d'années (ex.: en France, nouveaux types de collecteurs en plastique, mécanisation du détroquage du naissain et de la plupart des manipulations, extension des méthodes de culture en surélévation etc.). Or, malgré cet effort, l'ensemble des pays producteurs, sauf la Corée, subissent une stagnation, voire une régression de leur production (fig. 1). Quelles en sont les causes ?

En France, nous l'avons $\mathrm{vu}$, une catastrophe climatique et deux épizooties, l'une touchant la portugaise et l'autre la plate, expliquent les chutes et la stagnation globale de la production, malgré une forte demande intérieure. Les pollutions comme celles constatées dans le Bassin d'Arcachon ou, à la suite de l'échouage de l' "Amoco Cadiz», en Bretagne, sont aussi des facteurs de rếcession. A l'étranger on retrouve les mêmes causes pour expliquer les résultats : au Japon les pollutions, 


\section{mil1iers}

de

tonnes

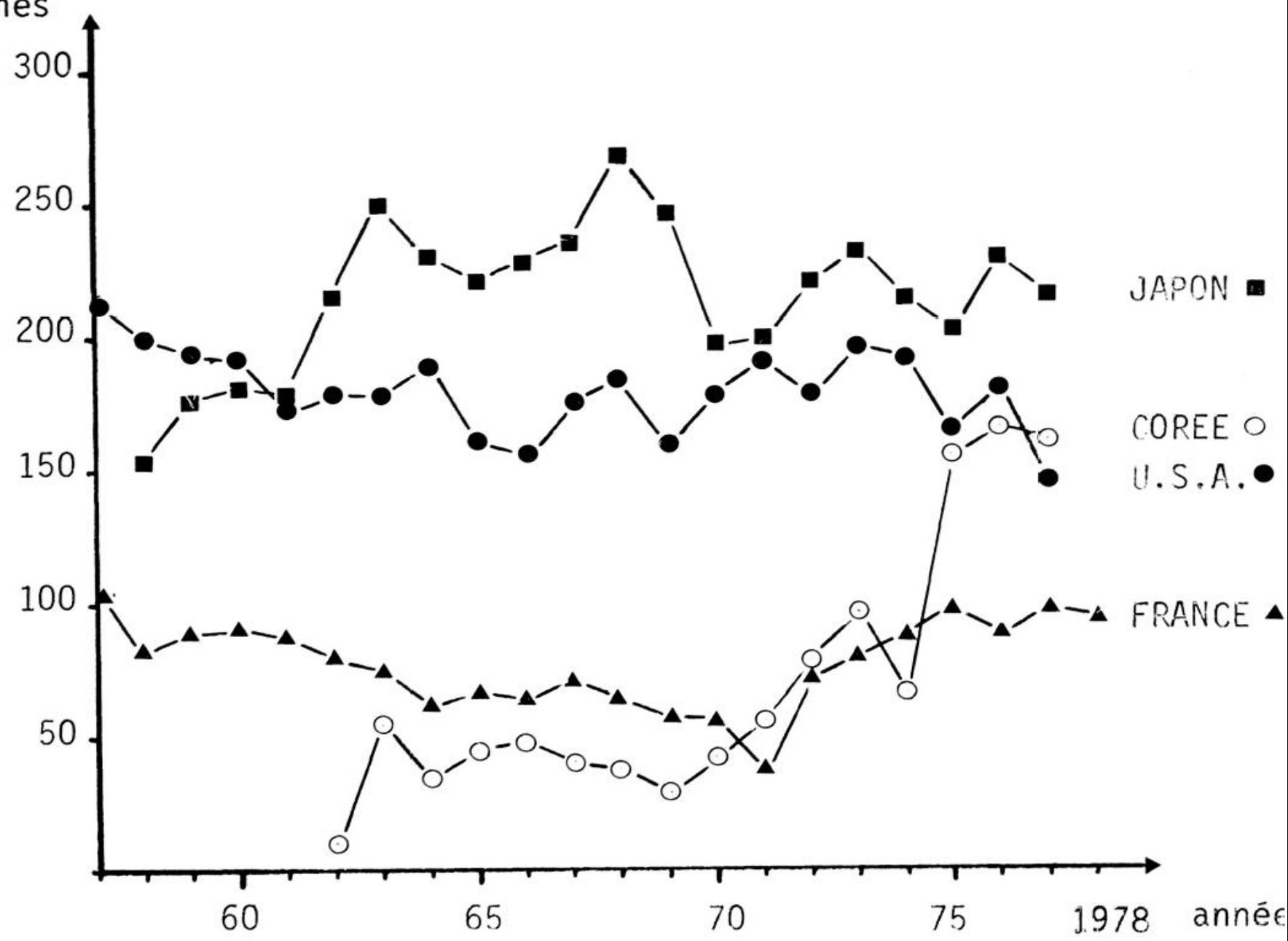

Fig. 1. - Production '"'uittres (plates et creuses) exprimée en poids brut (avec la coquille) drins les principaux pays ostréicoles du monde, depuis 1957

notamment celles de la Mer Intérieure, aux Etats-Unis une épizootie, le MSX. Dans aucun cas, une quelconque saturation du marché n'est invoquée, bien au contraire.

\section{B) LES MOULES.}

La moule, Mytilus edulis, est négligée en Amérique et refusée au Japon. En effet, le Japonais, qui est friand de tous les produits de la mer, y compris les animaux les plus ináttendus comme les méduses, les ascidies et les holothuries, ne consomme pas les moules, pourtant commune sur ses côtes.

La mytiliculture, née en Europe, est longtemps demeurée son exclusivité. Mais depuis une quinzaine d'années, elle est devenue une branche active de l'aquaculture coréenne. 


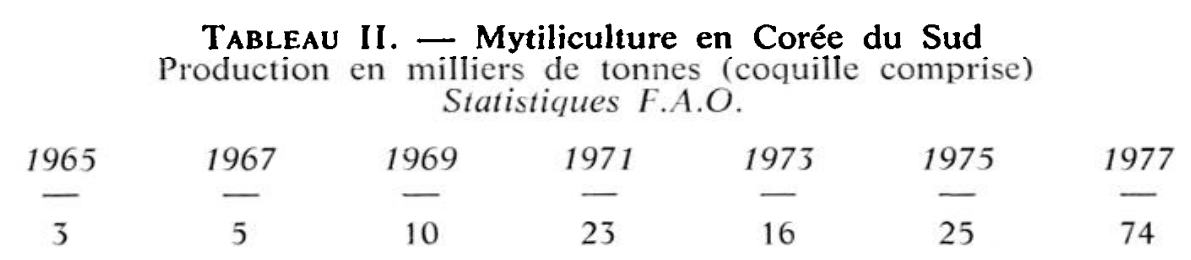

La production de moules en Europe est relativement stable depuis quelques années.

\begin{tabular}{|c|c|c|c|c|c|c|c|c|c|c|}
\hline & Proc & TA & en $\mathrm{I}$ & $\begin{array}{l}\text { ers } \\
\text { atist }\end{array}$ & $\begin{array}{l}\text { tilicu } \\
\text { tonn } \\
\text { ies } F \text {. }\end{array}$ & $\begin{array}{l}\text { re e } \\
\text { (coc } \\
O .\end{array}$ & $\begin{array}{l}\text { Europ } \\
\text { Ie co }\end{array}$ & prise & & \\
\hline & & $F$ & $D K$ & $G$ & $\operatorname{IRL}$ & $G B$ & $N L$ & $S P$ & $I$ & Total \\
\hline & & - & - & 一 & - & - & - & - & - & - \\
\hline 1975 & $\ldots \ldots \ldots \ldots \ldots$ & 55 & 28 & 17 & 4 & 7 & 112 & 92 & 4 & 319 \\
\hline 1976 & $\ldots \ldots \ldots \ldots$ & 44 & 38 & 23 & 4 & 7 & 73 & 65 & 5 & 259 \\
\hline 1977 & $\ldots \ldots \ldots \ldots$ & 53 & 47 & 11 & 4 & 11 & 109 & 61 & 4 & 300 \\
\hline
\end{tabular}

C'est en France que l'on trouve les méthodes de cultures les plus diversifiées. Ainsi, la production de 1975 provenait de trois sources dans les proportions suivantes: bouchots $75 \%$, sur cordes $13 \%$, cultures à plat $12 \%$. La culture à plat est la seule qui soit employée en Hollande et dans les pays nordiques. La culture en suspension, qui nécessite des infrastructures coûteuses, telles que des pilotis (ex. Bassin de Thau) ou des radeaux (ex : Ria Aroza en Espagne) est répandue en Méditerranée (Italie, France, pour 6.000 t) et surtout dans les rias de Galice, qui fournissent la quasi-totalité de la production espagnole. Les bouchots, qui consistent en des pieux verticaux sur lesquels sont enroulées les cordes de moules, se rencontrent surtout en France où leur présence a profondément modifié l'aspect de l'estran en Charente et, plus récemment, en Bretagne nord.

\section{C) LES PECTINIDES.}

La coquille Saint-Jacques du genre Pecten et les pétoncles du genre Chlamys sont les plus connus des Pectinidés. Pour une espèce, le Patinopecten yessoensis, une méthode de culture a été mise au point au Japon à partir de 1961. Malgré son caractère récent, nous la classons dans la conchyliculture traditionnelle car cette "pectiniculture» est établie sur les mêmes bases que l'ostréicuture et la mytiliculture : le captage du naissain dans le milieu naturel. Quant aux méthodes de cultures, elles sont aussi très variées : soit à plat en eau profonde, soit en suspension sur cordes où les animaux sont attachés par les " oreilles » de leur coquille, ou placés dans des paniers en filet de modèles divers. Cette activité qui s'exerce dans la partie nord du Japon (baie de Mutsu, lac Saroma et baie de Uchiura) est en progrès constant (tabl. IV). Cependant des mortalités massives liées à la surcharge des lieux d'élevage ont été enregistrées ces dernières années. 


$$
\begin{aligned}
& \text { Tableau IV. - Pectiniculture au Japun } \\
& \text { Production en milliers de tonnes (coquille comprise) } \\
& \text { Statistiques du ministère de l'Agriculture du Japon }
\end{aligned}
$$

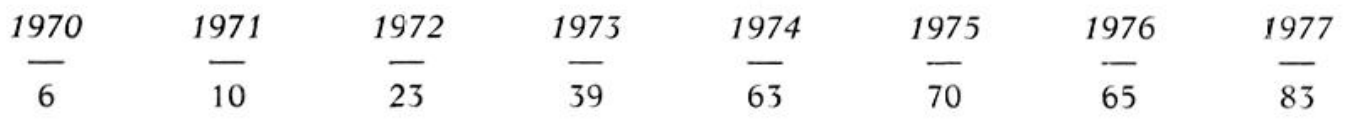

Les mêmes méthodes de captage ont été tentées sur Pecten maximus, la coquille Saint-Jacques européenne, en France, en Irlande et en GrandeBretagne. Les meilleurs résultats ont été obtenus en baie de Saint-Brieuc par le Centre Océanologique de Bretagne (C.N.E.X.O.), mais les résultats sont encore trop irréguliers pour envisager une réelle culture.

\section{D) Les Algues.}

Les algues sont cultivées depuis fort longtemps en Extrême-Orient, où elles servent à l'alimentation humaine.

C'est le nori, Porphyra spp. et notamment $P$. japonica, une algue rouge consommée séchée, qui a donné lieu aux premiers essais de culture au Japon à la fin du $\mathrm{XvI}^{\mathbf{e}}$ siècle. Il s'agissait d'offrir des supports adéquats aux algues (fascines, piquets, cordes). Au $\mathrm{xx}^{\mathfrak{e}}$ siècle, les progrès de la biologie ont permis de découvrir puis de maîtriser le cycle complet du nori (spore-conchochelis-thalle). Désormais, les aquaculteurs produisent eux-mêmes leurs spores en salle, soit collectivement dans le cadre d'une coopérative, soit même individuellement, sans avoir besoin d'une infrastructure particulièrement lourde ou coûteuse. Aussi, la distinction entre aquaculture traditionnelle et aquaculture nouvelle est-elle très difficile à établir.

Actuellement, le nori est l'algue la plus couramment produite, au

\begin{tabular}{|c|c|c|c|c|c|c|c|c|}
\hline \multicolumn{9}{|c|}{ 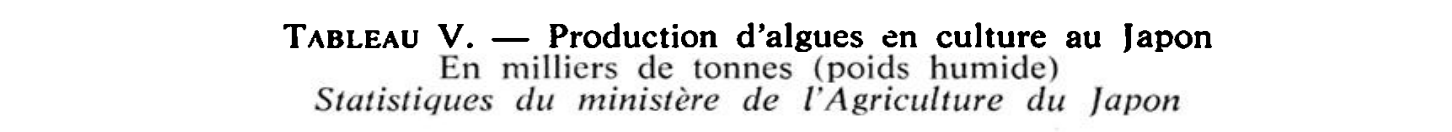 } \\
\hline & 1970 & 1971 & 1972 & 1973 & 1974 & 1975 & 1976 & 1977 \\
\hline & - & - & - & - & - & - & - & \\
\hline Nori $\ldots$ & 231 & 245 & 218 & 311 & 339 & 278 & 291 & 279 \\
\hline Wakame ...... & 76 & 95 & 106 & 113 & 154 & 102 & 127 & 126 \\
\hline Laminaire $\ldots \ldots$ & & & 3 & 8 & 10 & 16 & 22 & 27 \\
\hline
\end{tabular}
Japon (voir tabl. V), mais aussi en Chine (150.000 $t$ en 1973) et en Corée du Sud (180.000 t en 1973).

Outre la production des spores, la seconde « révolution » technique en noriculture consiste en la généralisation, au cours des vingt dernières années, de la conservation à basse température des filets chargés d'algues. Ce stockage, qui peut durer quelques jours ou quelques semaines, permet d'une part d'éviter les catastrophes climatiques (dessalure brutale, températures trop élevées) et pathologiques qui y sont souvent liées. D'autre part, au moment de la récolte et de la collecte des spores, cette technique permet un meilleur étalement des opérations dans le temps, donc une production plus forte. 
Enfin, au Japon et en Corée, la mécanisation du travail se développe rapidement : récolte à l'aide d'aspirateurs, broyage, séchage et transformation du produit à l'aide de machines spécialisées.

Malgré toutes ces améliorations, il s'est produit un net fléchissement de la production japonaise de nori depuis 1974 (tabl. V). Il s'agit d'un problème de marché, la production de $339.000 \mathrm{t}$ enregistrée alors étant nettement excédentaire, surtout en produits de qualité médiocre. Depuis, les efforts ont porté sur l'amélioration qualitative du nori et la sélection de souches et d'espèces supérieures, tandis que la reconversion des aquaculteurs dans la pisciculture marine était encouragée.

Nous signalons ici les cultures d'algues brunes, bien qu'en toute rigueur, elles appartiennent aux " cultures nouvelles », car à la différence du nori, leur production ne s'est développée que récemment grâce aux progrès scientifiques et technologiques. Le wakame Undaria pinnatifida et les laminaires Laminaria spp. sont au Japon des espèces prometteuses pour l'aquaculture. Les structures nouvelles résistantes aux tempêtes (palangres, filières) ont permis l'installation de champs suspendus de laminaires, au large, dans le nord du Japon, dont l'exploitation est rentable. Le wakame, cultivé dans des eaux plus chaudes, est concurrencé par d'autres élevages, mais la production augmente régulièrement (tabl. V). Les algues brunes sont aussi cultivées en Chine (150.000 t de laminaires en 1973) et en Corée du Sud (160.000 t d'Undaria en 1973).

\section{II. - L'AQUACULTURE NOUVELLE.}

Elle a quatre caractéristiques : elle se base sur des données scientifiques souvent récentes ; elle réalise des élevages complets, à partir de l'œuf ; elle s'applique à toutes sortes d'espèces; elle se veut industrielle et non pas artisanale.

Pour l'instant, malgré d'énormes efforts publics ou privés, cette aquaculture nouvelle n'a que rarement franchi le stade expérimental ou pré-industriel. Nous examinerons successivement la situation dans les secteurs les plus avancés : les mollusques, les crustacés, les poissons.

\section{A) Les mollusoues.}

La conchyliculture nouvelle réalise la production de naissain en écloserie. Dans une écloserie, l'eau de mer est filtrée, thermostatée et sa qualité est contrôlée. A partir de géniteurs qui ont subi un conditionnement, on provoque la ponte induite et les embryons obtenus sont élevés dans des conditions écologiques précises et, nourris avec des algues monocellulaires de culture, passent par différents stades larvaires, puis se métamorphosent en postlarves. On obtient ainsi du petit naissain (de l'ordre du millimètre) libre de tout support. Après un prégrossissement en nurserie, qui est une installation moins sophistiquée que la 
précédente, le naissain atteint une taille (de l'ordre du centimètre) comparable au naissain détroqué capté dans la nature. Il pourra donc subir ultérieurement les mêmes méthodes de culture. Ainsi, le naissain d'écloserie peut s'intégrer aux cultures classiques de mollusques.

Cette technique a d'énormes avantages: elle s'applique à toutes les espèces, y compris à celles dont on ne sait pas faire le captage en mer (ormeaux, palourdes...) ; elle permet de fournir du naissain en fonction de la demande et à toute époque de l'année. Elle a cependant un grave défaut : elle est extrêmement coûteuse. Dès lors on peut se demander si les écloseries industrielles qui existent à travers le monde sont rentables.

Au Japon, les écloseries ne fournissent massivement qu'un mollusque, l'ormeau (Haliotis discus); les pêcheurs groupés en coopératives les achètent et les placent en mer sur des récifs artificiels. Ils les pêchent quand ils sont adultes. En fonction du prix élevé de l'ormeau au Japon, l'opération serait rentable à tous les niveaux.

Les écloseries des pays occidentaux (plusieurs aux Etats-Unis, quelquesunes en France, Grande-Bretagne, Espagne et Irlande) sont surtout spécialisées dans la fourniture du naissain de bivalves : les huîtres $O$. edulis et $C$. gigas, les Palourdes Venerupis decussata (l'européenne) et $V$. semidecussata (la japonaise) et, à moindre échelle, le clam Mercenaria mercenaria. Leur rentabilité n'est pas évidente. Le plus souvent, les écloseries sont aidées par l'Etat, mais dans certains cas, ce sont des firmes industrielles qui prennent le risque d'entretenir ces établissements, dans l'espoir de pouvoir dominer un jour le marché du naissain de bivalves... Les causes actuelles de non-rentabilité sont diverses; il faut beaucoup de personnel, la maîtrise technique n'est pas entièrement acquise et les ventes aux éleveurs ne sont pas régulières. Malgré ces difficultés, l'activité des écloseries ne cesse de s'accroître, en particulier au niveau de cultures nouvelles, comme celle de la palourde en France.

\section{B) Les CRustacés.}

Les crustacés sont caractérisés par leurs nombreux stades larvaires et leur croissance par mues, qui sont autant de périodes critiques pour l'animal : leur élevage est donc délicat. Mais la haute valeur marchande des crustacés et la raréfaction dans la nature des espèces les plus recherchées ont suscité de gros efforts d'investigation de la part des scientifiques.

Pour certaines espèces, il s'agit seulement d'obtenir des post-larves et de les lâcher dans le milieu naturel dans le but d'un " repeuplement ". Ainsi, depuis le XIX ${ }^{e}$ siècle, des lâchers de bébés-homards ont été réalisés, sans qu'on ait pu démontrer une incidence sur les captures d'adultes dans les mêmes zones quelques années après. En dépit de cette incertitude, l'aquaculture de repeuplement se poursuit dans quelques pays dont la France. 
Pour d'autres crustacés, dont le cycle de développement est court, l'élevage complet a été réalisé. Au Japon, la crevette Penaeus japonicus est produite par des organismes semi-publics et par des éleveurs commerciaux. Malgré le poids de l'investissement (écloserie, bassins extérieurs de grande dimension), ces élevages s'avèrent rentables et la production augmente (tabl. VI) du fait d'un meilleur contrôle des épizooties.

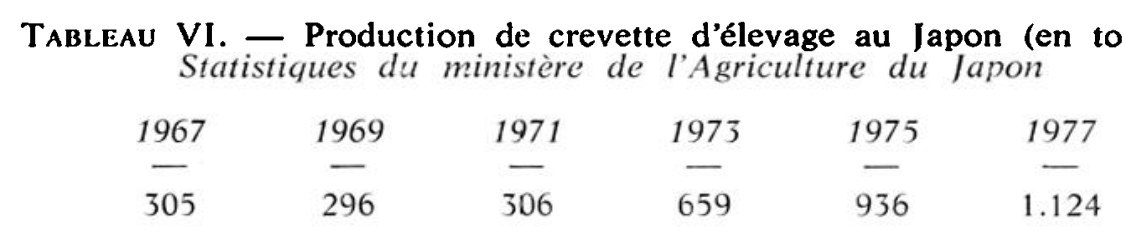

Par contre, l'élevage du crabe bleu Neptunus trituberculatus a été abandonné après quelques années (11 t en 1967, 15 en 1968, 5 en 1974 et rien depuis 1975).

C) LES POISSONS.

$\mathrm{Au}$ Japon sont cultivés commercialement les sérioles : Seriola quinqueradiata et $S$. purpurescens, les daurades, notamment Chrysophrys major, les chinchards : Trachurus japonicus et $T$. delicatissimus, l'alutère Stephanolepis cirrhifer et le fugu Fugu rubripes.

Le chinchard est élevé en grossissement, principalement comme appât pour la pêche au thon. Les autres espèces sont destinées à la consommation humaine, et particulièrement la consommation crue pour la sériole et la daurade. La daurade, en outre, est élevée dans le but du repeuplement en Mer Intérieure de Seto. Nous ne parlerons pas ici de l'anguille et du mulet, considérées comme espèces d'eau douce. La sériole et la daurade sont des poissons de mers chaudes élevés dans le sud du Japon. Les juvéniles sont pêchés en mer puis placés dans des cages flottantes de filet, où ils sont nourris de poissons et coquillages jusqu'à atteindre la taille optimale en un, deux ou trois ans. La production augmente régulièrement (tabl. VII). La demande dépasse l'offre pour tous les poissons " de luxe ", ce qui explique que les élevages de fugu, très difficiles, se relèvent après avoir presque disparu. Les difficultés techniques et les investissements nécessaires à la pisciculture marine ne découragent pas les aquaculteurs, car c'est une branche profitable, étant clonné le prix élevé du produit.

TABleau VII. - Production de poissons marins au Japon (en tonnes) Statistiques du ministère de l'Agriculture du Japon

\begin{tabular}{|c|c|c|c|c|c|c|c|c|}
\hline & 1970 & 1971 & $\underline{1972}$ & 1973 & $\begin{array}{c}1974 \\
-\end{array}$ & $\begin{array}{c}1975 \\
-\end{array}$ & 1976 & $\begin{array}{c}1977 \\
\end{array}$ \\
\hline Chinchard & 7 & 57 & 127 & 378 & 667 & 942 & 762 & 904 \\
\hline Sériole ......... & 43.354 & 61.855 & 77.059 & 80.439 & 92.946 & 92.407 & 101.786 & 115.038 \\
\hline Daurade ....... & 454 & 930 & 1.380 & 2.741 & 3.298 & 4.462 & 6.572 & 8.245 \\
\hline Fugu $\ldots \ldots \ldots$ & 23 & 15 & 14 & 16 & 8 & 9 & 11 & 15 \\
\hline Alutère $\ldots . .$. & 62 & 18 & 39 & 40 & 25 & 8 & 2 & 10 \\
\hline
\end{tabular}


Les Salmonidés sont cultivés en Europe et en Amérique du Nord. Le cycle naturel du saumon est de se reproduire en rivière et de s'engraisser en mer. On peut soumettre la truite à ce régime, qui a pour avantage une meilleure croissance et une chair plus appréciée. Les pisciculteurs d'eau douce ont maîtrisé depuis longtemps les techniques de ponte et d'alevinage. Le passage en eau de mer est une phase délicate de l'élevage, sur laquelle se concentrent les efforts des chercheurs, qui ont aussi à résoudre des problèmes d'alimentation et de pathologie.

L'élevage en mer se fait soit dans des enclos, soit dans des cages flottantes. La production commerciale de saumon de l'Atlantique (Salmo salar) et de truite de mer a débuté vers 1970 en Norvège, qui reste en tête (100 $\mathrm{t}$ en $1971,12.000 \mathrm{t}$ en 1979 dont 4.000 de saumon). Les saumons du Pacifique (espèces du genre Oncorhynchus) sont élevés aux E.U. (500 t en 1979). En France, les élevages portent sur la truite et le saumon du Pacifique (200 t en 1979).

Parallèlement à l'élevage complet, on pratique une sorte de « repeuplement " avec les Salmonidés, en utilisant le fait que chaque individu, après engraissement libre en mer, revient, pour la reproduction, dans sa rivière d'origine. C'est la technique du sea-ranching très employée sur les côtes américaines du Pacifique avec les Oncorhynchus et plus modérément en Irlande avec le saumon atlantique.

\section{III. - LES CONDITIONS D'UN ESSOR DE LA MARICULTURE.}

\section{A) L'ESPACE EN ZONE LITTORALE.}

Le littoral, zone de contact et d'échanges est devenu depuis peu, zone de croissance, donc lieu de convoitise. Dès lors, les activités d'aquaculture, qui sont forcément localisées à la frange côtière, se heurtent à d'autres intérêts: les ports, les industries, le tourisme, l'urbanisation. Toutes ces activités, avides d'espace, entrent en concurrence. Examinons le cas de la France : la conchyliculture y a débuté il y a un siècle, aussi les sites propices ont-ils été convertis en concessions, mais des méthodes nouvelles sont apparues récemment : où placer les écloseries et les bassins d'élevage sur ce littoral devenu " patrimoine exigu et précaire »? Les professionnels conscients de ce conflit, effectif ou potentiel, ont alerté les Pouvoirs publics. Dans certains cas, ils ont été entendus. Ainsi, dans trois départements bretons, un inventaire des sites favorables à l'aquaculture a été dressé à la demande des autorités départementales, qui ont pris des dispositions pour les réserver à cet usage. Mais cette initiative, déjà tardive, est loin d'être générale. Il est indubitable que l'essor de la mariculture en France sera limité par le manque d'espace. 
B) La qualité de L'EAu de mer.

La qualité de l'eau, quasiment constante en haute mer est très souvent dégradée sur le littoral. En effet, l'eau côtière est soumise non seulement aux effets des activités industrielles, agricoles et urbaines qui s'exercent in situ, mais aussi aux effets des pollutions lointaines, d'origine terrestre (par l'intermédiaire des fleuves) ou, plus rarement, marine (hydrocarbures par exemple).

Les effets des agents polluants y sont de deux sortes : soit mortalité ou affaiblissement des élevages, soit insalubrité des produits de consommation par suite du phénomène de bioaccumulation (de métaux lourds, de pesticides ou de bactéries et virus pathogènes). La parade à cette situation est le contrôle sanitaire : dans certains cas, on peut épurer les produits contaminés, dans d'autres cas, il faut les détruire. En certains endroits de la Baltique ou de la Mer du Nord, toutes les cultures marines sont devenues impossibles en raison d'un excès de pollution. Ce phénomène est une menace très grave pour l'avenir de l'aquaculture.

\section{C) LES CONNAISSANCES TECHNOLOGIQUES ET SCIENTIFIQUES.}

Le matériel utilisé pour les cultures marines est soumis à une corrosion intense et, lorsqu'il est placé en pleine eau, à des efforts mécaniques très rudes. L'usage récent du plastique a permis l'essor d'une technologie adaptée à ces contraintes, mais il s'agit d'un marché encore rudimentaire. Aussi, le plus souvent, les aquaculteurs doivent avoir recours à du matériel artisanal qu'ils ont eux-mêmes élaboré avec plus ou moins de bonheur. La pauvreté technologique est souvent une cause d'échec en aquaculture.

Les problèmes biologiques qui se posent dans les cultures marines sont de trois ordres : la pathologie, l'écophysiologie, la génétique. Nous avons signalé les ravages des épidémies sur les cultures d'huîtres : le problème est d'autant plus grave que l'isolement, la "quarantaine ", des animaux touchés en milieu marin est impossible, la mer jouant le rôle de vecteur. L'écophysiologie, c'est-à-dire le fonctionnement des organismes en rapport avec les facteurs du milieu, est peu avancée pour le milieu marin, qui est très complexe. Les échecs de beaucoup d'écloseries s'expliquent par une insuffisance des connaissances écophysiologiques de l'espèce cultivée. Quant à la génétique, qui a fait le succès de l'agriculture, tant pour les plantes que pour les animaux, elle est encore très rudimentaire pour les espèces marines.

Toutes ces considérations nous montrent que les problèmes de technologie et de biologie sont plus difficiles à résoudre en mer que dans les autres milieux; leur solution est une des conditions de l'essor de l'aquaculture. 


\section{D) L'EXEMPLE JAPONAIS.}

Les résultats du Japon en mariculture sont exceptionnels, à la fois au vu des tonnages produits $(861.000 \mathrm{t}$ en 1977) et de la diversité des cultures. Beaucoup de secteurs sont en pleine expansion (coquille SaintJacques, laminaire, sériole), la recherche se poursuit activement et s'oriente vers l'aquaculture au large des côtes.

Parmi les raisons qui expliquent le succès économique de la mariculture japonaise, il faut citer le besoin et le goût des Japonais pour les produits de la mer, autrement dit l'existence d'un marché important et exigeant. La tradition établie précocément par les ostréiculteurs et les noriculteurs a permis aux communautés du littoral d'acquérir une expérience irremplaçable, en même temps qu'une organisation sociale qui ont servi d'assises à l'aquaculture moderne. Le gouvernement, les chercheurs, les autorités locales travaillent en étroite collaboration avec les aquaculteurs regroupés en coopératives ou fédérations bien structurées. Ces raisons socio-économiques s'ajoutent à l'avantage géographique du Japon, riche en baies, estuaires, lagunes favorables à l'aquaculture, baigné par un courant froid l'Oya-shio et un courant chaud le Kuro-shio, donc doté d'une faune marine abondante et diverse.

Tant qu'il demeurait mal connu, l'océan, qui recouvre $71 \%$ de la surface du globe, passait pour une source inépuisable de vivres. On est revenu de cette illusion quand les stocks halieutiques ont pu être sérieusement évalués, car on s'est alors rendu compte que les ressources océaniques n'étaient pas illimitées. Cependant, durant les années 1960, les quantités pêchées annuellement ne cessaient de crôitre, et les limites semblaient très élevées. Ainsi, en 1971, M. La Prairie, alors directeur du C.N.E.X.O., résumait l'opinion générale : «On constate une sous-exploitation des océans, car, si en 1968 les quantités pêchées s'élevaient à 60 millions de tonnes, on estime en fait à 200 millions de tonnes les possibilités réelles de capture ». Aujourd'hui, les experts inclinent plutôt pour un maximum de 100 millions de tonnes. Quant à la production réelle, elle stagne depuis près d'une décennie, puisque les statistiques de la F.A.O. évaluaient à 62,7 millions de tonnes le total pêche et aquaculture en 1977. Ces constats conduisent à une évidence: la pêche ne résoudra pas le problème de la faim dans le monde.

Plus tard, les espoirs se sont portés sur l'aquaculture. Là encore, on imaginait que les possibilités étaient infinies. Or, nous avons vu que les tonnages produits par la mariculture n'ont progressé depuis vingt ans $(2,2$ millions de tonnes en 1977) que grâce aux algues cultivées en Extrême-Orient ( 1 million de tonnes en 1977). Une analyse des causes de cette stagnation a montré que le manque d'espace, les difficultés 
techniques et surtout les ravages des épidémies et des pollutions contrarient les efforts des hommes en ce domaine. Certes, on constate quelques réussites localisées: la progression spectaculaire de la production de mollusques en Corée du Sud, la diversification des espèces cultivées au Japon. Des progrès ont aussi été accomplis, notamment au niveau des techniques nouvelles, en Europe et aux Etats-Unis. Mais si l'on s'en tient aux résultats concrets, force est de constater que la mariculture ne progresse que très lentement. Ce n'est pas elle non plus qui, dans les prochaines années, apportera les ressources alimentaires qui manquent à l'humanité.

En définitive, le souhait le plus raisonnable que l'on puisse faire est que les productions marines (pêche et aquaculture) progressent dans le même rapport que les productions continentales, c'est-à-dire qu'elles continuent à représenter $3 \%$ de la nourriture mondiale et $11 \%$ des protéines consommées. Dans ce but, l'effort en faveur des cultures marines doit se poursuivre, car, comme l'a écrit Amanieu (in Bougis et coll., 1974) : «L'aquaculture n'apparaît plus comme un choix hasardeux, mais comme une réalité dont il convient d'assurer le succès et non de flatter ou de minimiser les performances. »

\section{REFERENCES BIBLIOGRAPHIQUES}

F.A.O. Statistiques - Pêches - Captures et quantités débarquées. Vol. 34 (1972), vol. 38 (1974), vol. 44 (1977).

France. Statistiques annuelles de la Marine marchande, de 1957 à 1978.

Japon. Annuaire statistique sur la pêche et l'aquaculture, 1977. Ministère de l'Agricultu:e, des forêts et des pêches. Tokyo, 1979 (en japonais).

ANONYME, 1974 : Perspectives pour l'aménagement. Littoral français. Rapport au Gouvernement, novembre 1973. La Documentation Française, Paris, $268 \mathrm{p}$.

Bougis et coll., 1976: Océanographie biologique appliquée. L'exploitation de la vie marine, Masson, Paris, 320 p.

Doumenge (F.), 1975: Actualités de la pêche et de l'aquaculture au Japon. Société Languedocienne de Géographie, Montpellier.

LA Prairie (Y.), 1971 : La conquête du nouvel espace. In 2000 . « Le 6c continent».

LuCAS (N.), 1976 : L'aquaculture, in Encyclopédie permanente du Japon, vol. 1, Presses Orientalistes de France, Paris.

\section{RESUME}

Les productions marines par culture pouvaient être évaluées en 1977 à 2,2 millions de tonnes (dont 1 million d'algues) tandis que les captures par pêche s'élevaient à 60,5 millions. La mariculture est en pleine évolution technologique et s'est considérablement diversifiée dans les vingt dernières années, notamment au Japon et, dans une moindre mesure, en Europe et aux Etats-Unis. Cependant les tonnages annuels de l'aquaculture dans les mers tempérées, ne progressent guère depuis vingt ans, sauf dans certains secteurs au Japon et en Corée du Sud. Les causes de cette stagnation sont surtout les épidémies et les pollutions qui font chuter les cultures traditionnelles, le manque d'espace et les difficultés techniques qui gênent la progression des nouvelles. 


\section{SUMMARY}

Marine culture, in the year 1977, can be evaluated at 2.2 millions tons (1 million tons for algae), while the catches by fishing amounted to 60.5 millions tons. Mariculture is in full technical evolution and was considerably diversified in the last twenty years, especially in Japan and, to a lesser degree, in Europe and U.S.A. However the annual production of aquaculture in temperate seas did not increase during the last twenty years, except in some areas in Japan and South Korea. The causes of this stagnation were on one hand epidemies and pollutions which determined a decrease in traditional cultures, on the other hand the lack of space and underdevelopment in technics which retard the progress of new cultures. 